\title{
Pegylated liposomal-paclitaxel induces ovarian cancer cell apoptosis via TNF-induced ERK/AKT signaling pathway
}

\author{
ZHIYING QI, LIRONG YIN, YANYING XU and FANG WANG
}

Department of Gynaecology and Obstetrics, The Second Hospital of Tianjin Medical University, Tianjin 300211, P.R. China

Received March 30, 2017; Accepted December 5, 2017

DOI: $10.3892 / \mathrm{mmr} .2018 .8811$

\begin{abstract}
Ovarian cancer is one of the most common gynecological types of cancer and is characterized by a relatively high incidence and high mortality rate. Evidence has demonstrated that paclitaxel (PTX) is an effective therapeutic treatment for human ovarian cancer. In the present study, the inhibitory effects of pegylated liposomal (PL)-PTX on the growth of ovarian cancer cells were investigated in vitro; a CAOV-3-bearing mouse model was established to investigate the in vivo effects of PL-PTX on ovarian tumor growth. In the present study, the underlying mechanism of tumor necrosis factor (TNF)-induced inhibition of extracellular signal-regulated kinase $(\mathrm{ERK}) /$ protein kinase B (AKT) signaling pathway mediated by PL-PTX was analyzed within ovarian cancer cells. The results of the present study revealed that PL-PTX significantly inhibited the growth and aggressiveness of ovarian cancer cells in vitro and in vivo and apoptotic ability increased upon administration of PL-PTX. The expression levels of caspase-3/9 were significantly upregulated within PL-PTX-treated ovarian cancer cells. The expression and phosphorylation levels of ERK and AKT were markedly increased in response to PL-PTX treatment. In addition, the inhibitory effects of PL-PTX on ovarian cancer cells were eliminated by neutralizing antibodies against TNF. The observations of the present study revealed that PL-PTX induced ovarian cell apoptosis via the TNF-dependent pathway, which was significantly inhibited with the employment of antibodies against TNF. In vivo analysis demonstrated that PL-PTX treatment significantly inhibited ovarian tumor growth and prolonged the survival of tumor bearing mice. In conclusion, the findings of the present study have provided an insight into the potential
\end{abstract}

Correspondence to: Professor Lirong Yin, Department of Gynaecology and Obstetrics, The Second Hospital of Tianjin Medical University, 23 Longchang Road, Hexi, Tianjin 300211, P.R. China

E-mail: yinlirongtianjin@163.com

Key words: pegylated liposomal-paclitaxel, ovarian tumor, apoptosis, tumor necrosis factor, extracellular signal-regulated kinase/protein kinase B mechanism of PL-PTX-induced apoptosis of ovarian cancer cells. As PL-PTX has been reported to induce ovarian tumor cell apoptosis via the TNF-induced ERK/AKT signaling pathway, PL-PTX may serve as an efficient anticancer drug for the treatment of ovarian cancer.

\section{Introduction}

Ovarian cancer exhibits high rates of mortality compared with other gynecological malignances (1). Investigations into the pathogenesis of ovarian cancer have reported that diagnosis occurs within the later stages of pathogenesis, which has been associated with lower overall survival rates $(2,3)$. Previous studies have reported that ovarian cancer metastasizes throughout the peritoneal cavity and numerous organs may be affected during the pathogenesis of ovarian cancer $(4,5)$. At present, the incidence rate of ovarian cancer is increasing; omental metastases have been observed in $80 \%$ of patients with severe ovarian cancer (6). Without effective treatment, cases of ovarian cancer may progress to mortality. Therefore, effective treatment may improve the prognosis of ovarian cancer $(7,8)$.

Paclitaxel (PTX) is a tricyclic diterpene compound that may be delivered from poly-(DL-lactic-co-glycolic) acid or pegylated liposomal (PL) foams for controlled release in postoperative chemotherapy against glioblastoma multiforme (9). PTX exhibits anticancer properties in patients with epithelial ovarian cancer (10). In addition, PTX reveals therapeutic effects in the treatment of human cancers, including ovarian, breast, lung, colorectal, melanoma, head and neck cancer, lymphoma and brain tumors (11-13). Furthermore, antiangiogenic therapy vs. dose-dense PTX therapy for the frontline treatment of epithelial ovarian cancer has been reviewed in phase III randomized clinical trials (14). Notably, the development and evaluation of the novel tocopheryl methoxy poly (ethylene glycol)-block-(lactic-co-glycolic acid) copolymer nanoparticles significantly enhance the therapeutic effects of PTX for the treatment of ovarian cancer (15).

In the present study, the efficacy of PL-PTX was investigated within ovarian cancer cells and a tumor-bearing mouse model. Treatment with PL-PTX inhibited the growth and aggressiveness of ovarian cancer cells, and significantly induced ovarian cancer cell apoptosis in vitro and in vivo. Additionally, the apoptotic signaling pathways mediated by PL-PTX within ovarian cancer cells were investigated. 


\section{Materials and methods}

Ethics statement. The present study was conducted with the recommendations in the guide for the Care and Use of Laboratory Animals and was approved by the ethics committee of The Second Hospital of Tianjin Medical University, Tianjin, China (approval no. 20151105DGB). All animal protocols were conducted and maintained in accordance with the National Institutes of Health and approved by the ethics committee of Animal Experiments Defence Research of the Second Hospital of Tianjin Medical University (Tianjin, China; Approval validity: Oct 2015 to Oct 2017).

Cell culture. CAOV-3 cells were obtained from the American Type Culture Collection (Manassas, VA, USA). All cells were cultured in Dulbecco's modified Eagle's medium (Thermo Fisher Scientific, Inc., Waltham, MA, USA) supplemented with $10 \%$ fetal bovine serum (Thermo Fisher Scientific, Inc.). All cells were cultured in a $37^{\circ} \mathrm{C}$ humidified atmosphere containing $5 \% \mathrm{CO}_{2}$.

MTT assay. CAOV-3 cells ( $1 \times 10^{3}$ cells/well) were incubated with PTX $(5,10$ and $15 \mathrm{mg} / \mathrm{ml})$ or PL-PTX $(5,10$ and $15 \mathrm{mg} / \mathrm{ml})$ in 96-well plates for 24,48 and $72 \mathrm{~h}$ at $37^{\circ} \mathrm{C}$ in triplicate for each condition, with PBS serving as a control. Following incubation, $20 \mu 1$ MTT ( $5 \mathrm{mg} / \mathrm{ml})$ in PBS was added to each well and the plate was further incubated for $4 \mathrm{~h}$. The majority of the medium was removed and $100 \mu 1$ dimethyl sulfoxide was added into the wells to solubilize the crystals. Optical density was measured with an ELISA reader (Bio-Rad Laboratories, Inc., Hercules, CA, USA) at a wavelength of $570 \mathrm{~nm}$.

Cells invasion and migration assays. CAOV-3 cells $\left(1 \times 10^{5}\right.$ cells/well) were cultured at $37^{\circ} \mathrm{C}$ in an environment containing $5 \% \mathrm{CO}_{2}$ until $90 \%$ confluence was attained. CAOV-3 cells were then incubated with PTX $(10 \mathrm{mg} / \mathrm{ml})$, PL-PTX $(10 \mathrm{mg} / \mathrm{ml})$ or extracellular signal-regulated kinase (ERK) inhibitor (cat. no. ab142271; Abcam, Cambridge, UK) treatment for $48 \mathrm{~h}$ at $37^{\circ} \mathrm{C}$. For the invasion assay, $1 \times 10^{5}$ CAOV-3 cells were suspended in $500 \mu 1$ serum-free RPMI (Thermo Fisher Scientific, Inc.) and $500 \mu 1$ RPMI with 5\% FBS (Thermo Fisher Scientific, Inc.) was ploaced in the lower chamber for $48 \mathrm{~h}$ at $37^{\circ} \mathrm{C}$. The cells were plated in the upper chambers of BD BioCoat Matrigel Invasion Chambers (BD Biosciences, Franklin Lakes, NJ, USA) according to the manufacturer's protocol. Cotton swabs were used to remove cells and to plate cells. To investigate migration, CAOV-3 cells $\left(1 \times 10^{6}\right)$ were cultured in RPMI medium in the upper chamber and RPMI medium with 5\% FBS (Thermo Fisher Scientific, Inc.) in the lower chamber. Cells were then plated in a control insert chamber (BD Biosciences) for $48 \mathrm{~h}$ at $37^{\circ} \mathrm{C}$. Cells were stained using $0.1 \%$ crystal violet dye (Sigma-Aldrich; Merck KGaA) for $30 \mathrm{~min}$ at $37^{\circ} \mathrm{C}$. Tumor cell migration and invasion were observed in $\geq 3$ randomly-selected stained-fields per membrane under a light microscope (BX51; Olympus Corporation, Tokyo, Japan).

Western blot analysis. CAOV-3 cells were then incubated with PTX (10 mg/ml), PL-PTX (10 mg/ml) or ERK inhibitor (cat. no. ab142271; Abcam) for $48 \mathrm{~h}$ at $37^{\circ} \mathrm{C}$. Cells were collected and lysed in radioimmunoprecipitation assay buffer (M-PER reagent for cells and T-PER reagent for tissues; Thermo Fisher Scientific, Inc.) followed by homogenization at $4^{\circ} \mathrm{C}$ for $10 \mathrm{~min}$. Protein concentration was measured with a bicinchoninic acid protein assay kit (Thermo Fisher Scientific, Inc.). A total of $20 \mu \mathrm{g}$ protein was electrophoresed via $12.5 \%$ SDS-PAGE and subsequently transferred to nitrocellulose membranes. Prior to incubation with primary antibodies at $4^{\circ} \mathrm{C}$ overnight, membranes were incubated with blocking buffer (5\% milk) for $2 \mathrm{~h}$ at $37^{\circ} \mathrm{C}$. The primary antibodies used were against: Caspase-9 (1:1,200; ab32539), ERK (1:1,000; ab54230) and phosphorylayed (p)ERK 1/2 (phospho-Thr202/Tyr204; 1:1,000; ab214362), caspase-3 (1:1,200; ab2171), protein kinase B (AKT; 1:1,000; ab8805), p-AKT (phosphor-S473; 1:1,000; ab8932) and $\beta$-actin (1:500; ab8226; all Abcam) for $12 \mathrm{~h}$ at $4^{\circ} \mathrm{C}$. Horseradish peroxidase-conjugated anti-rabbit immunoglobulin $\mathrm{G}(\mathrm{IgG}$; Bio-Rad Laboratories, Inc.) was applied to membranes at a 1:5,000 dilution for $2 \mathrm{~h}$ at $37^{\circ} \mathrm{C}$ and detected using an enhanced chemiluminescence substrate ECL Select ${ }^{\mathrm{TM}}$ (Roche Diagnostics, Basel, Switzerland). The density of the protein bands was analyzed by Quantity One software version 4.62 (Bio-Rad Laboratories, Inc.).

Apoptosisassay. CAOV-3 cells $\left(5 \times 10^{6}\right)$ were then incubated with PTX $(10 \mathrm{mg} / \mathrm{ml})$, PL-PTX $(10 \mathrm{mg} / \mathrm{ml})$ or and or ERK inhibitor (ERKIR; IR7936) and/or anti-TNF $\alpha$ (1:1,000; cat. no. ab6671; $2 \mathrm{mg} / \mathrm{ml}$; both Abcam) treatment for $48 \mathrm{~h}$ at $37^{\circ} \mathrm{C}$. Following trypsinization, cells were washed in cold PBS and adjusted to $1 \times 10^{6}$ cells/ml using PBS 3 times at room temperature; CAOV-3 cells were labeled with Annexin V-fluorescein isothiocyanate (V-FITC) and propidium iodide (Annexin V-FITC kit, BD Biosciences) for $2 \mathrm{~h}$ at $37^{\circ} \mathrm{C}$, according to the manufacturer's protocol, and analyzed with a FACScan flow cytometer (BD Biosciences) using BD FACSChorus ${ }^{\mathrm{TM}}$ intuitively designed software version 1.2 (BD Biosciences).

Animal studies. Specific pathogen-free male Balb/c mice ( $\mathrm{n}=90 ; 8$ weeks old; $32-35 \mathrm{~g}$ body weight) were purchased from the Shanghai Laboratory Animal Center Co., Ltd. (Shanghai, China). All mice were housed separately and maintained in a $12 \mathrm{~h}$ light/dark cycle with $23 \pm 1^{\circ} \mathrm{C}$ with a relative and humidity of $50 \pm 5 \%$. All mice had free access to food and water. Nude mice were subcutaneously injected with CAOV-3 cells $\left(1 \times 10^{5}\right)$ into the right forelimb under aseptic conditions. All mice were housed in a temperature-controlled facility at $23 \pm 1^{\circ} \mathrm{C}$ with free access to food and water. Mice were randomly divided into three groups $(\mathrm{n}=30$ per group) and received a treatment of $10 \mathrm{mg} / \mathrm{kg}$ PTX, $10 \mathrm{mg} / \mathrm{kg}$ PL-PTX or PBS by intravenous injection. Treatments were initiated on day 3 following tumor implantation (diameter: 5-6 mm) and continued 10 times daily for a total of 20 days. Tumor volumes were calculated once every 3 days according to the formula: $V=0.5 x a^{2} x b$, where $a$ : Short diameter and $b$ : Long diameter of tumor as measured with a Vernier caliper.

Immunohistochemistry. Tumors from xenograft mice, extracted as previously described (16), were fixed by using $10 \%$ formaldehyde for $4 \mathrm{~h}$ at $37^{\circ} \mathrm{C}$ and embedded in paraffin (4- $\mu \mathrm{m}$ thick sections). Antigen retrieval was performed using a microwave to heat the sections (standard microwave settings; 20s) and graded series of ethanol, followed by 
A

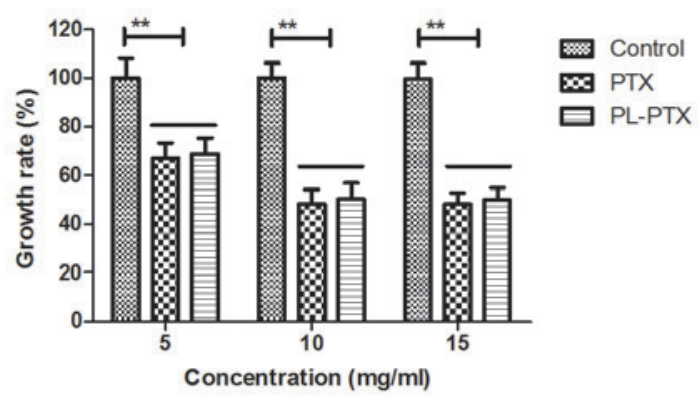

C

Control
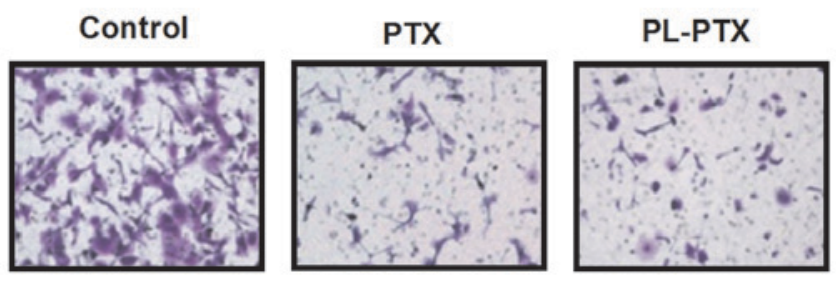

B
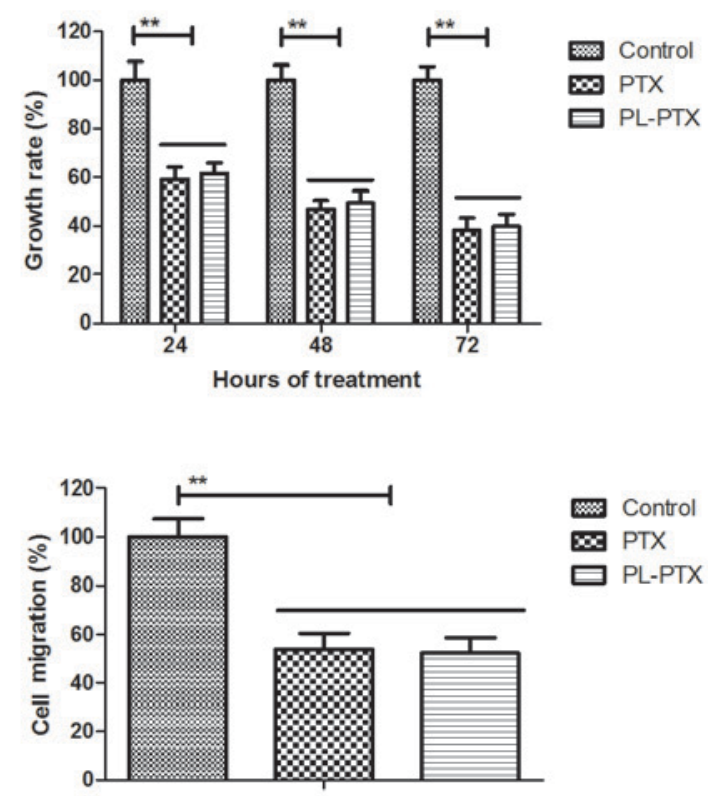

D
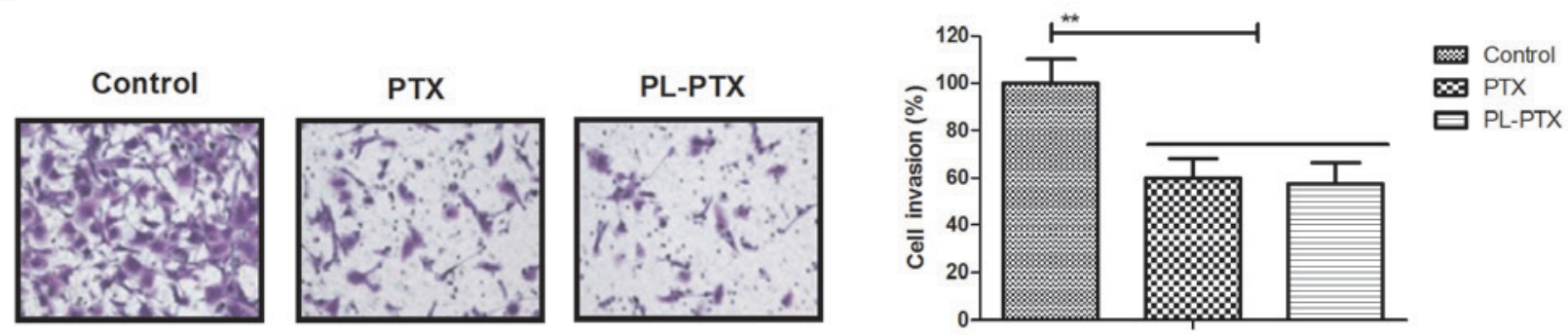

Figure 1. PL-PTX treatment inhibits the growth and metastasis of ovarian cancer cells. (A) PL-PTX and PTX inhibited ovarian cancer cell growth compared with control group. (B) PL-PTX inhibited the growth of ovarian cancer cells in a time-dependent manner compared with control group. PL-PTX (10 mg/ml) or PTX $(10 \mathrm{mg} / \mathrm{ml})$ suppressed ovarian cancer cell (C) migration and (D) invasion compared with the control group. Magnification, $\mathrm{x} 40 .{ }^{* *} \mathrm{P}<0.01 . \mathrm{PL}-\mathrm{PTX}$, pegylated liposomal-paclitaxel.

blocking of endogenous peroxidase activity with $3 \%$ hydrogen peroxide for $10 \mathrm{~min}$ at room temperature. Tumor sections were incubated with 5\% BSA (Sigma-Aldrich; Merck KGaA, Darmstadt, Germany) for $2 \mathrm{~h}$ at $37^{\circ} \mathrm{C}$ and then incubated with rabbit anti-mouse primary antibodies caspase-9 (1:1,200; ab32539) or caspase-3 (1:1,200; ab2171; both Abcam) for $12 \mathrm{~h}$ at $4^{\circ} \mathrm{C}$. Tumor tissues were washed with PBS three times and incubated with biotinylated secondary antibodies anti-rabbit IgG (1:2,000; Pierce; Thermo Fisher Scientific, Inc.) for $2 \mathrm{~h}$ at $37^{\circ} \mathrm{C}$. Biotin-peroxidase signals were detected using $0.5 \mathrm{mg} / \mathrm{ml}$ 3'3'-diaminobenzidine (DAB) $/ 0.003 \% \mathrm{H}_{2} \mathrm{O}_{2}$ (Dako; Agilent Technologies, Inc. Santa Clara, CA, USA) as a substrate. Results were recorded using a laser confocal microscope (BX51; Olympus Corporation).

Terminal deoxynucleotidyl transferase-mediated dUTP nick end labeling (TUNEL) assay. Apoptotic cells $\left(5 \times 10^{6}\right)$ of tumor specimens were fixed using $4 \%$ formaldehyd for $30 \mathrm{~min}$ at $37^{\circ} \mathrm{C}$ and analyzed using a TUNEL assay (DeadEnd ${ }^{\mathrm{TM}}$ Colorimetric TUNEL System; Promega Corporation, Madison, WI, USA) according to the manufacturer's protocol. Tumor sections were incubated with the reaction mixture (terminal deoxynucleotidyl transferase, equilibration buffer and biotinylated nucleotide mix) for $1 \mathrm{~h}$ at $37^{\circ} \mathrm{C}$. Subsequently, streptavidin- and DAB-bound biotin was quantified and counterstained with hemalum (1\%; Merck KGaA, Darmstadt, Germany) and aquatex (Merck $\mathrm{KGaA}$ ) for $1 \mathrm{~h}$ at $37^{\circ} \mathrm{C}$. Tumor sections were washed with PBS three times for $5 \mathrm{~min}$ at room temperature. DNA fragmentation was analyzed in 3 randomly selected fields of $4-\mu \mathrm{m}$ tumor sections using light microscope (magnification, $\mathrm{x} 40$ ).

Statistical analysis. Each experiment was performed at least three times. All data are expressed as the mean \pm standard deviation of triplicate dependent experiments and analyzed by using one-way analysis of variance followed by a Tukey test. All data were analyzed using SPSS software, version 19.0 (IBM Corp. Armonk, NY, USA), GraphPad Prism 5.0 (GraphPad Software, Inc., La Jolla, CA, USA) and Microsoft Excel (Microsoft Corporation, Redmond, WA USA). $\mathrm{P}<0.05$ and was considered to indicate a statistically significant difference.

\section{Results}

PL-PTX treatment inhibits the growth and aggressiveness of ovarian cancer cells. The inhibitory effects of PL-PTX on the growth and aggressiveness of ovarian cancer cells were studied 
A
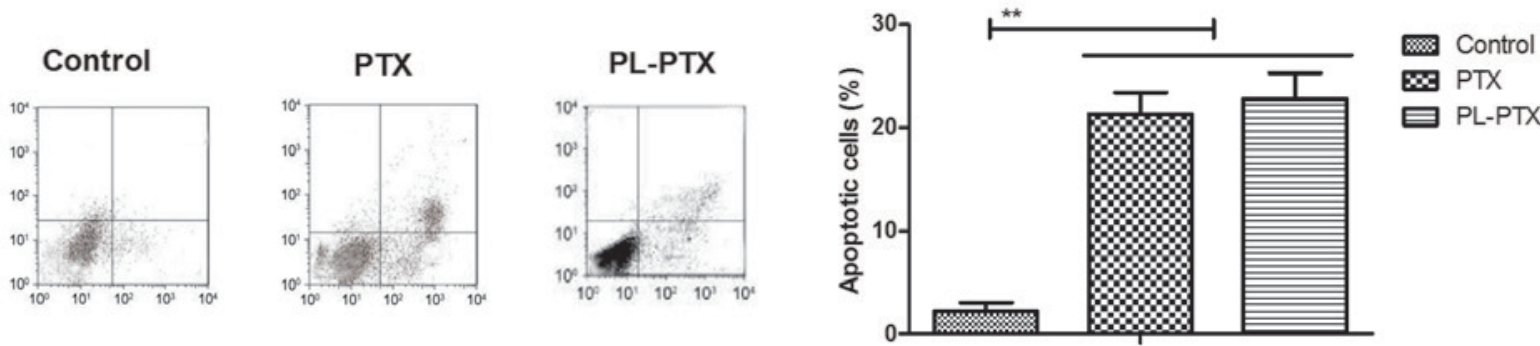

B

C
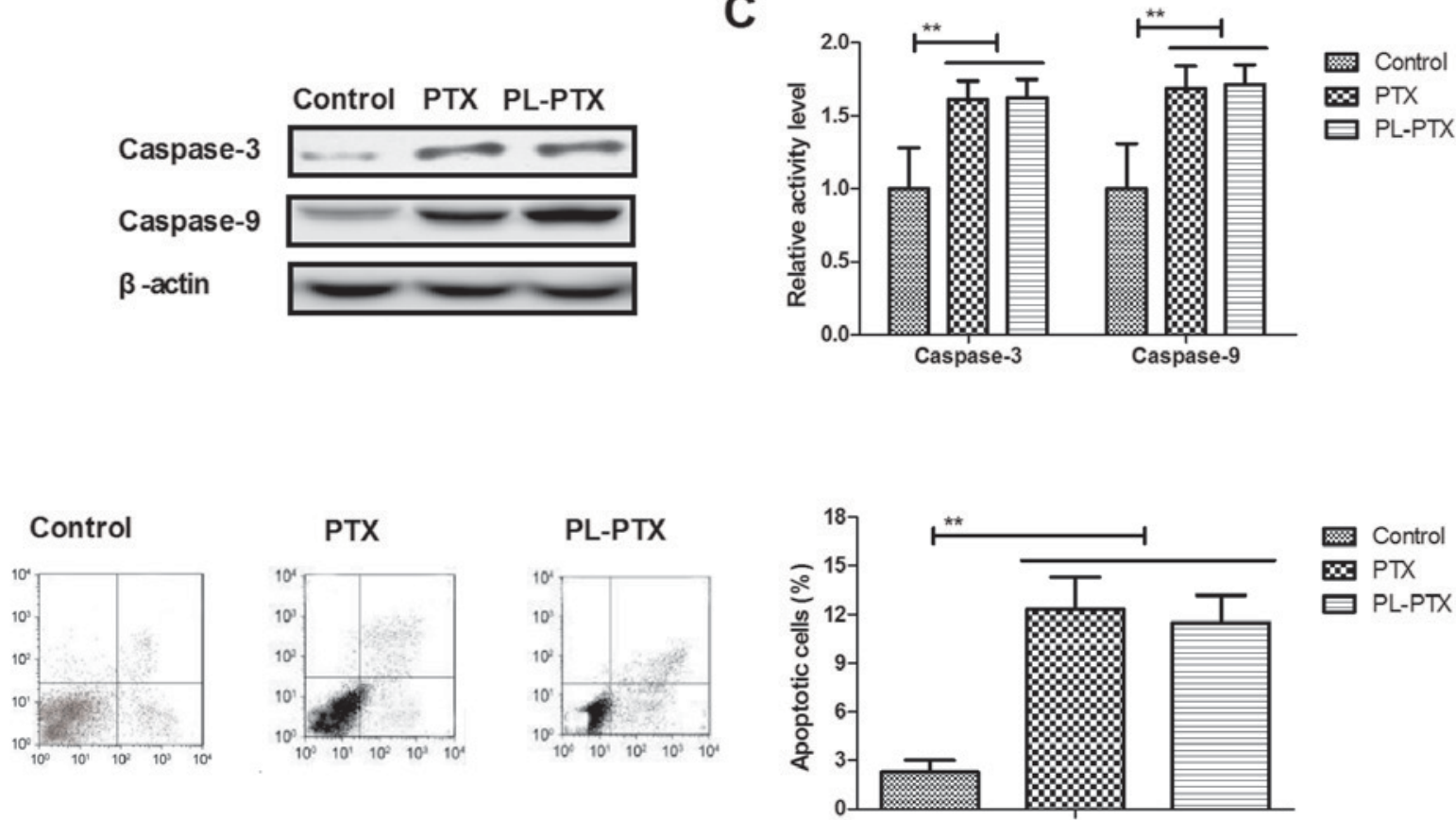

Figure 2. PL-PTX promotes ovarian cancer cell apoptosis via the caspase-dependent signal pathway. (A) PL-PTX promoted CAOV-3 ovarian cancer cell apoptosis compared with control group. (B) PL-PTX promoted caspase-3 and caspase-9 activity within CAOV-3 cells compared with control group. (C) PL-PTX treatment increased caspase- 3 and caspase-9 protein expression levels within CAOV-3 cells compared with control group. (D) Inhibition of caspase-3 activity via a pan-caspase inhibitor, partially decreased PTX- and PL-PTX-induced apoptosis of CAOV-3 cells compared with in the control group. ${ }^{* *} \mathrm{P}<0.01$. PL-PTX, pegylated liposomal-paclitaxel.

in vitro. PL-PTX and PTX treatment significantly inhibited ovarian cancer cell growth (Fig. 1A). In addition, PL-PTX and PTX inhibited growth of ovarian cancer cells in time-dependent manner (Fig. 1B). Treatments of $10 \mathrm{mg} / \mathrm{ml}$ PL-PTX or PTX significantly inhibited migration and invasiveness of ovarian cancer cells (Fig. 1C and D). The results of the present study indicated that PL-PTX significantly inhibited the growth and aggressiveness of ovarian cancer cells.

PL-PTX promotes apoptosis of ovarian cancer cells via the caspase-dependent signaling pathway. As presented in Fig. 2A, the apoptotic ability of CAOV-3 cells was promoted by PL-PTX. Significantly increased caspase- 3 and caspase- 9 activities were detected within PL-PTX-treated CAOV-3 cells (Fig. 2B). PL-PTX treatment increased caspase-3 and caspase-9 protein expression levels in CAOV-3 cells (Fig. 2C). These results demonstrated that PTX- and PL-PTX induced apoptosis of CAOV-3 cells (Fig. 2D). The results of the present study suggested that PL-PTX promoted ovarian cancer cell apoptosis via the caspase-dependent signaling pathway.
PL-PTX is associated with ovarian cancer cell apoptosis via tumor necrosis factor (TNF)-induced the ERK/AKT signaling pathway. TNF has been reported to promote tumor cell apoptosis $(17,18)$; the effects of TNF on PL-PTX-induced apoptosis were investigated in the present study. Employment of AntiTNF, an anti-TNF neutralizing antibody, partially reduced PTX- and PL-PTX-inhibited growth and aggressiveness of CAOV-3 cells (Fig. 3A-C). The results of the present study demonstrated that PL-PTX treatment led to a reduction in ERK and AKT expression and phosphorylation levels; however, anti-TNF inhibited the effects exhibited by PL-PTX (Fig. 3D and E). Additionally, inhibition of the TNF significantly reduced PTX- and PL-PTX-induced CAOV-3 cell apoptosis (Fig. 3F). These results suggested that PL-PTX may be associated with ovarian cancer metastasis, which is mediated by the TNF-induced inhibition of the ERK/AKT signaling pathway.

ERK/AKT signaling pathway is involved in the activation of the TNF/caspase-3 cascade within ovarian cancer cells. Associations between ERK/AKT and the caspase-3 cascade 
A

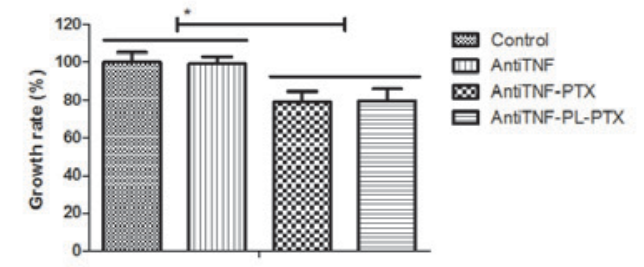

B
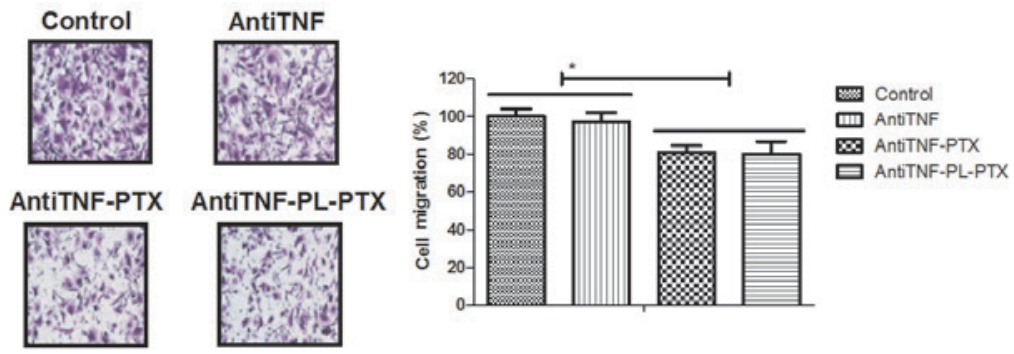

C

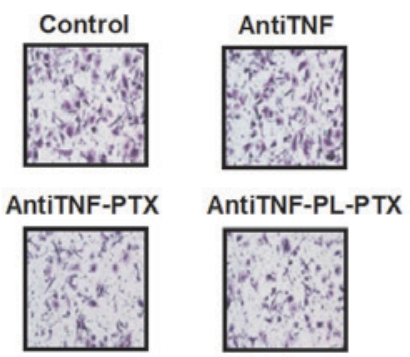

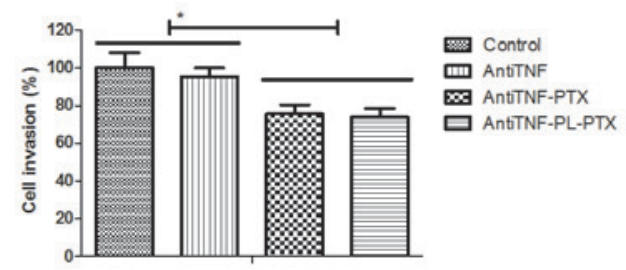

D

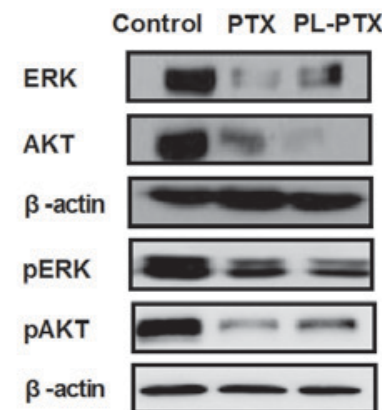

E

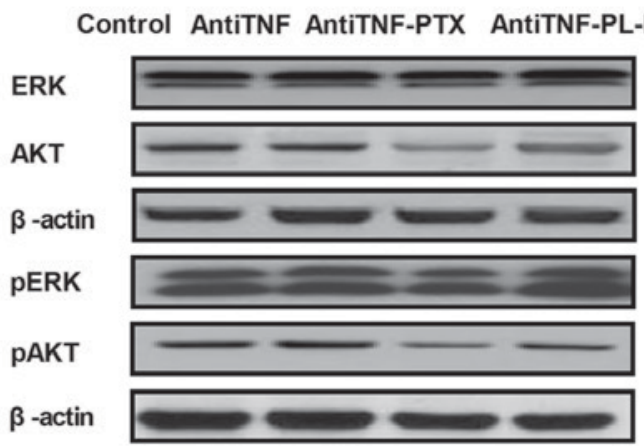

$\mathbf{F}$
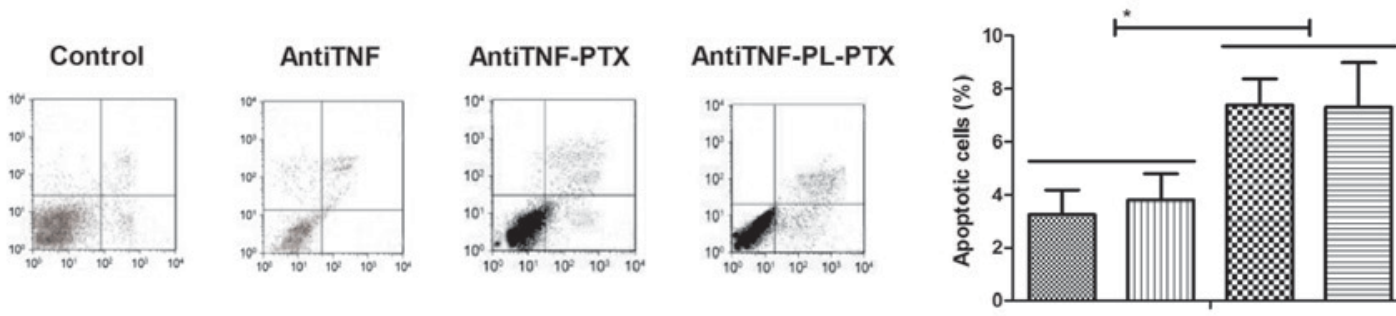

짐 Contro

띠 AntiTNF

B AntTNF-PTX

A AntiTNF-PL-PTX

Figure 3. PL-PTX is associated with ovarian cancer metastasis, mediated by tumor necrosis factor (TNF)-induced inhibition of the ERK/AKT signaling pathway. AntiTNF partially reduced PTX- and PL-PTX-inhibited (A) growth, (B) migration and (C) invasion of CAOV-3 cells compared with control group. (D) PL-PTX and PTX decreased ERK and AKT expression and phosphorylation levels within CAOV-3 cells compared with control group. (E) AntiTNF eliminated decreased ERK and AKT expression and phosphorylation levels induced by PTX and PL-PTX within CAOV-3 cells compared with control group. (F) Inhibition of ERK/AKT signal pathway reduced PTX- and PL-PTX-induced CAOV-3 cell apoptosis compared with control group. Magnification, $\mathrm{x} 40$. $^{*} \mathrm{P}<0.05$. AntiTNF, Anti-TNF neutralizing antibody. AKT, protein kinase B; ERK, extracellular signal-regulated kinase; PL-PTX, pegylated liposomalpaclitaxel; TNF, tumor necrosis factor.

within CAOV-3 cells were analyzed in the present study. The inhibition of ERK/AKT activity using ERKIR decreased the expression levels of caspase-3 within CAOV-3 cells compared with in cells control group (Fig. 4A). ERKIR inhibited
TNF-induced apoptosis of CAOV-3 cells compared with cells of the control group (Fig. 4B). PL-PTX-induced CAOV-3 cell apoptosis was also inhibited by an ERK inhibitor (Fig. 4C). The results of the present study suggested that the ERK/AKT 
A

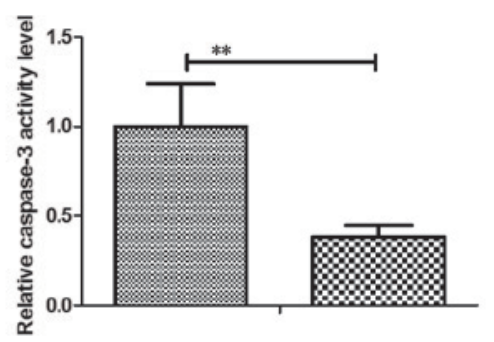

Control

$\infty$ ERKIR

B
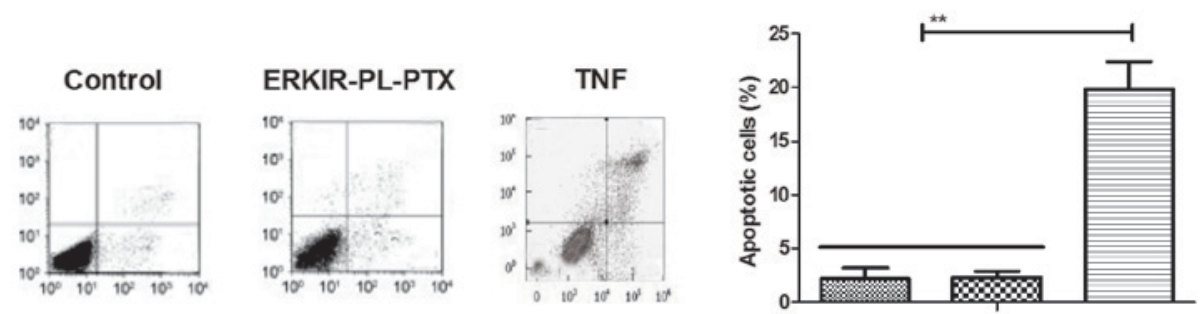

파 Control

$\mathbb{\infty}$ ERKIR-TNF

ㅁNF

C
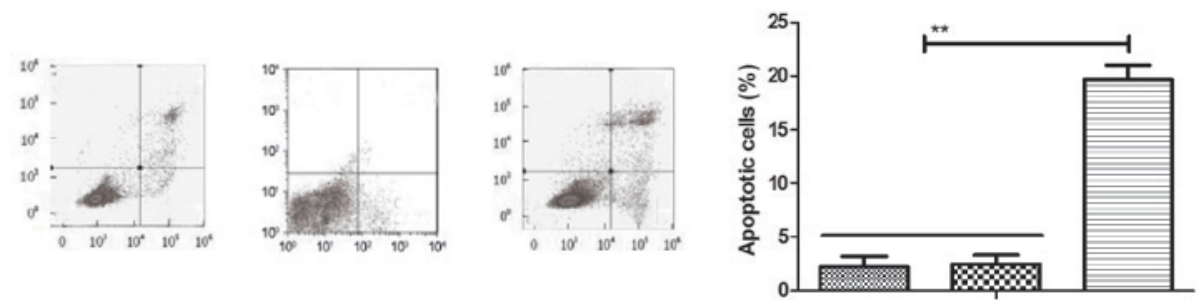

Control

E ERKIR-PL-PTX

曰 PL-PTX

Figure 4. ERK/AKT signaling pathway is involved in the activation of the TNF/caspase-3 cascade within ovarian cancer cells. (A) Inhibition of ERKIR decreased caspase-3 expression levels within CAOV-3 cells compared with in the control group. (B) Inhibition of ERKIR inhibited TNF-induced CAOV-3 cell apoptosis compared with in the control group. (C) PL-PTX-induced CAOV-3 cell apoptosis was inhibited via an ERK inhibitor compared with in the control group. ${ }^{* *} \mathrm{P}<0.01$. AKT, protein kinase B; ERK, extracellular signal-regulated kinase; ERKIR, ERK/AKT activity; PL-PTX, pegylated liposomal-paclitaxel; $\mathrm{TNF}$, tumor necrosis factor.

A

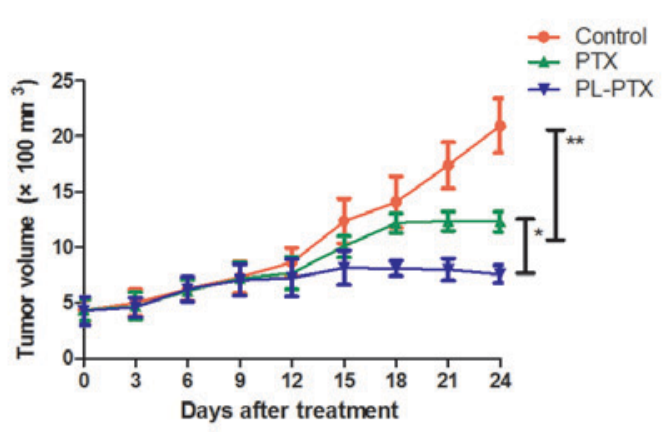

B

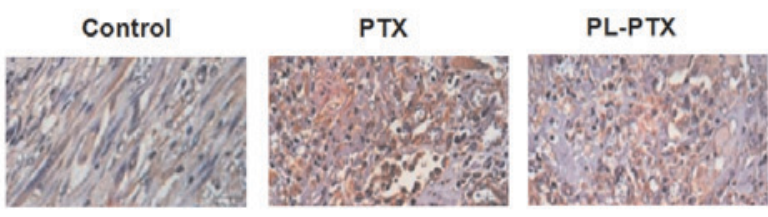

C

Control

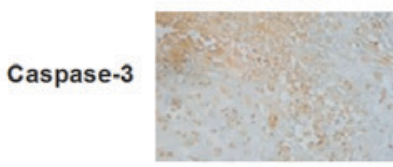

Caspase-9
PTX

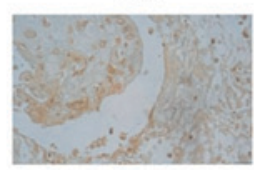

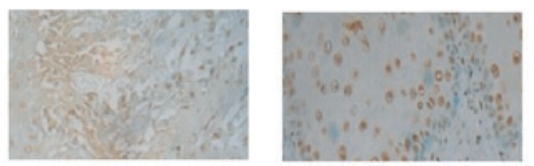

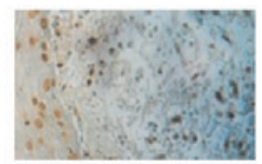

PL-PTX

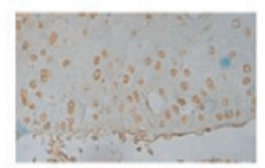

D

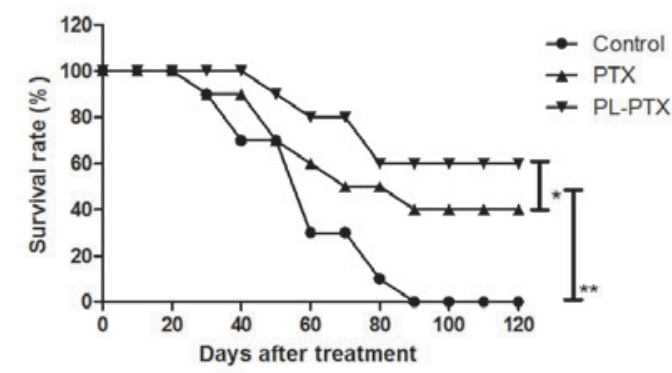

Figure 5. PL-PTX treatment suppresses in vivo growth of ovarian cancer cells within a tumor mouse model. (A) PL-PTX treatment significantly inhibited ovarian tumor growth compared with in the PTX and PBS groups in a $25 \mathrm{~d}$ observation. (B) PL-PTX treatment promoted tumor cell apoptosis compared with in the control group. (C) PL-PTX increased the expression levels of caspase-3 and caspase-9 within tumors compared with in the control group. (D) PL-PTX treatment prolonged survival of tumor bearing mice. Magnification, $\mathrm{x} 40 .{ }^{*} \mathrm{P}<0.05$ and ${ }^{* * *} \mathrm{P}<0.01$. PL-PTX, pegylated liposomal-paclitaxel. 
signaling pathway may be involved in the activation of the $\mathrm{TNF} /$ caspase-3 cascade within ovarian cancer cells.

PL-PTX treatment suppresses in vivo growth of ovarian cancer cells within a tumor mouse model. CAOV-3-bearing mouse model was established and received treatment of PL-PTX (10 mg/kg), PTX (10 mg/kg) or PBS once a day. In vivo analyses revealed that PL-PTX and PTX treatments significantly inhibited the growth of ovarian cancer cells compared with cells of the PBS groups in a 20 day observation (Fig. 5A). TUNEL analysis revealed that PL-PTX treatment significantly promoted tumor cell apoptosis compared with in cells of the PTX and PBS group (Fig. 5B). Caspase-3 and caspase-9 expression levels were upregulated in response to PL-PTX and PTX treatments (Fig. 5C). In addition, prolonged survival was observed within the PL-PTX treated group compared with in the PTX and PBS treated groups (Fig. 5D).

\section{Discussion}

Ovarian cancer has been associated with poor prognosis despite the administration of maximal multimodal therapy (19). Patients with advanced ovarian cancer are frequently diagnosed with metastatic cancer $(20,21)$. It has previously been demonstrated that PTX exerts anticancer properties on human malignancies by inducing apoptosis and inhibiting tumor cell growth and proliferation (22-24). A systematic review indicated that PL-PTX is more efficient compared with PTX in inhibiting growth and tumor metastasis of advanced, recurrent or refractory types of ovarian cancer (25). In the present study, the efficacy of PL-PTX within ovarian cancer cells was analyzed in vitro and in vivo; PL-PTX treatment was associated with the suppression of growth, migration and invasiveness, and promotion of apoptosis of ovarian cancer cells.

The tolerance of weekly metronomic PTX and carboplatin have been regarded as neoadjuvant chemotherapies for patients with advanced ovarian cancer; the efficacy of weekly vs. every-3-week administration of PTX has been compared in patients with ovarian cancer $(26,27)$. Compared with PTX, PL-PTX exhibits increased efficiency against the growth and aggressiveness of ovarian cancer cells. Perkins et al (28) demonstrated that PTX upregulates the protein expression levels of apoptotic peptidase activating factor-1, caspase-9, and BH3-interating domain death agonist during the mitochondrial events of apoptosis. The results of the present study indicated that PL-PTX treatment upregulated caspase-3 expression levels within ovarian cancer cells; a previous study reported that PTX treatment induces apoptosis of anaplastic thyroid cancer cells via caspase-3 activation (29). The findings of the present study suggested that PL-PTX treatment promoted ovarian cancer cell apoptosis via a caspase-dependent signaling pathway.

Previously, recombinant TNF- $\alpha$ has been demonstrated to be beneficial in patients with epithelial ovarian cancer receiving PTX and cisplatinum (30). In the present study, PL-PTX treatment was associated with the suppression of ovarian cancer via activation of the TNF-caspase-3 cascade within ovarian cancer cells. Suyama et al (31) reported that
ERK activation and retinoblastoma protein phosphorylation may serve as markers of PTX sensitivity of lung adenocarcinoma cells. In addition, previous studies have demonstrated PTX-induced apoptosis of human gastric cancer cells via inhibition of the ERK/AKT signaling pathway (32). In the present study, PTX induced ovarian cancer cell apoptosis via the induction of the TNF-mediated downregulation of ERK/AKT signaling pathway; a previous study proposed the association of the ERK/AKT signaling activation with cancer cell-resistance to PTX treatment (33). Additionally, a previous report demonstrated that upregulation of caspase-3 expression levels inhibits lung cancer metastasis and migration in a protease-independent manner via the downregulation of the ERK signaling pathway (34). The present study suggested that the ERK/AKT signaling pathway is involved in the activation of the TNF/caspase-3 cascade via PL-PTX within ovarian cancer cells.

In conclusion, analysis of the potential mechanism of PL-PTX-induced apoptosis within ovarian cancer cells revealed that PL-PTX may serve as an efficient anticancer drug in the treatment of ovarian cancer. In addition, the data of the present study demonstrated the potent apoptotic and anti-metastatic roles exhibited by PL-PTX in the treatment of ovarian cancer. Therefore, PL-PTX is more efficient compared with PTX in inhibiting tumor growth in vivo by mediating the ERK/AKT signaling inhibited by TNF.

\section{Acknowledgements}

Not applicable.

\section{Funding}

No funding was received.

\section{Availability of data and materials}

The analyzed data sets generated during the study are available from the corresponding author on reasonable request.

\section{Authors' contributions}

ZQ performed experiments. LY designed this study. YX and FW analyzed all data in the present study.

\section{Ethics approval and consent to participate}

The present study was conducted with the recommendations in the guide for the Care and Use of Laboratory Animals and was approved by the ethics committee of The Second Hospital of Tianjin Medical University, Tianjin, China (approval no. 20151105DGB).

\section{Consent for publication}

Not applicable.

\section{Competing interests}

The authors declare that they have no competing interests. 


\section{References}

1. Tempfer CB, El Fizazi N, Ergonenc H and Solass W: Metastasis of ovarian cancer to the breast: A report of two cases and a review of the literature. Oncol Lett 11: 4008-4012, 2016.

2. Shakeel S, Elit L, Akhtar-Danesh N, Schneider L and Finley C: Care delivery patterns, processes, and outcomes for primary ovarian cancer surgery: A Population-based review using a national administrative database. J Obstet Gynaecol Can 39: 25-33, 2017.

3. Yamamoto A, Miyasaka Y,Furuya K, Watanabe H, Maruyama M, Nakada H, Takano A, Hada M, Nakagomi H, Omata M and Oyama T: Pseudo-Meigs' syndrome due to ovarian metastases from colon cancer: A case report and review of the literature. Surg Case Rep 2: 112, 2016.

4. Pimentel C, Becquet M, Lavoue V, Henno S, Leveque J and Ouldamer L: Ovarian metastases from breast cancer: A series of 28 cases. Anticancer Res 36: 4195-4200, 2016.

5. Lago V, Minig L and Fotopoulou C: Incidence of lymph node metastases in apparent Early-stage low-grade epithelial ovarian cancer: A comprehensive review. Int J Gynecol Cancer 26 1407-1414, 2016

6. Raave R, de Vries RB, Massuger LF, van Kuppevelt TH and Daamen WF: Drug delivery systems for ovarian cancer treatment: A systematic review and meta-analysis of animal studies. PeerJ 3: e1489, 2015.

7. Munhoz RR, Pereira AA, Sasse AD, Hoff PM, Traina TA, Hudis CA and Marques RJ: Gonadotropin-releasing hormone agonists for ovarian function preservation in premenopausal women undergoing chemotherapy for Early-stage breast cancer: A systematic review and Meta-analysis. JAMA Oncol 2: 65-73, 2016.

8. Bian C, Yao K, Li L, Yi T and Zhao X: Primary debulking surgery vs. neoadjuvant chemotherapy followed by interval debulking surgery for patients with advanced ovarian cancer. Arch Gynecol Obstet 293: 163-168, 2016.

9. Ong BY, Ranganath SH, Lee LY, Lu F, Lee HS, Sahinidis NV and Wang $\mathrm{CH}$ : Paclitaxel delivery from PLGA foams for controlled release in post-surgical chemotherapy against glioblastoma multiforme. Biomaterials 30: 3189-3196, 2009.

10. Ansaloni L, Coccolini F, Morosi L, Ballerini A, Ceresoli M, Grosso G, Bertoli P, Busci LM, Lotti M, Cambria F, et al: Pharmacokinetics of concomitant cisplatin and paclitaxel administered by hyperthermic intraperitoneal chemotherapy to patients with peritoneal carcinomatosis from epithelial ovarian cancer. Br J Cancer 112: 306-312, 2015.

11. Karmakar S, Banik NL and Ray SK: Combination of all-trans retinoic acid and paclitaxel-induced differentiation and apoptosis in human glioblastoma U87MG xenografts in nude mice. Cancer 112: 596-607, 2008

12. Nikanjam M, Gibbs AR, Hunt CA, Budinger TF and Forte TM: Synthetic nano-LDL with paclitaxel oleate as a targeted drug delivery vehicle for glioblastoma multiforme. J Control Release 124: 163-171, 2007

13. Merighi S, Benini A, Mirandola P, Gessi S, Varani K, Leung E, Maclennan S, Baraldi PG and Borea PA: Hypoxia inhibits paclitaxel-induced apoptosis through adenosine-mediated phosphorylation of bad in glioblastoma cells. Mol Pharmacol 72: 162-172, 2007.

14. Slaughter KN, Moore KN and Mannel RS: Anti-angiogenic therapy versus dose-dense paclitaxel therapy for frontline treatment of epithelial ovarian cancer: Review of phase III randomized clinical trials. Curr Oncol Rep 16: 412, 2014.

15. Lv W, Cheng L and Li B: Development and evaluation of a novel TPGS-mediated paclitaxel-loaded PLGA-mPEG nanoparticle for the treatment of ovarian cancer. Chem Pharm Bull (Tokyo) 63: 68-74, 2015.

16. Lai J, Cai Q, Biel MA, Wang C, Hu X, Wang S and Lin J: Id1 and NF-kB promote the generation of CD133+ and BMI-1+ keratinocytes and the growth of xenograft tumors in mice. Int J Oncol 44: $1481-1489,2014$.

17. Griffith TS, Anderson RD, Davidson BL, Williams RD and Ratliff TL: Adenoviral-mediated transfer of the TNF-related apoptosis-inducing ligand/Apo-2 ligand gene induces tumor cell apoptosis. J Immunol 165: 2886-2894, 2000.
18. Sato T, Yamauchi N, Sasaki H, Takahashi M, Okamoto T, Sakamaki S, Watanabe N and Niitsu Y: An apoptosis-inducing gene therapy for pancreatic cancer with a combination of 55-kDa tumor necrosis factor (TNF) receptor gene transfection and mutein TNF administration. Cancer Res 58: 1677-1683, 1998.

19. Yang XJ, Zheng FY, Xu YS and Ou RY: Ovarian cancer initially presenting with isolated ipsilateral superficial inguinal lymph node metastasis: A case study and review of the literature. J Ovarian Res 7: 20, 2014.

20. Debniak T, Gromowski T, Scott RJ, Gronwald J, Huzarski T, Byrski T, Kurzawski G, Dymerska D, Górski B, Paszkowska-Szczur K, et al: Management of ovarian and endometrial cancers in women belonging to HNPCC carrier families: Review of the literature and results of cancer risk assessment in Polish HNPCC families. Hered Cancer Clin Pract 13: 3, 2015.

21. Ebell MH, Culp M, Lastinger K and Dasigi T: A systematic review of the bimanual examination as a test for ovarian cancer. Am J Prev Med 48: 350-356, 2015.

22. Han X, Chen J, Jiang M, Zhang N, Na K, Luo C, Zhang R, Sun M, Lin G, Zhang R, et al: Paclitaxel-paclitaxel prodrug nanoassembly as a versatile nanoplatform for combinational cancer therapy. ACS Appl Mater Interfaces 8: 33506-33513, 2016.

23. Fukuchi M, Mochiki E, Ishiguro T, Ogura T, Sobajima J, Kumagai Y, Ishibashi K and Ishida H: Efficacy of Nab-paclitaxel as Second-line chemotherapy for unresectable or recurrent gastric cancer. Anticancer Res 36: 6699-6703, 2016.

24. Zhang T, Luo J, Fu Y, Li H, Ding R, Gong T and Zhang Z: Novel oral administrated paclitaxel micelles with enhanced bioavailability and antitumor efficacy for resistant breast cancer. Colloids Surf B Biointerfaces 150: 89-97, 2017.

25. Edwards SJ, Barton S, Thurgar E and Trevor N: Topotecan, pegylated liposomal doxorubicin hydrochloride, paclitaxel, trabectedin and gemcitabine for advanced recurrent or refractory ovarian cancer: A systematic review and economic evaluation. Health Technol Assess 19: 1-480, 2015.

26. Narod SA: Weekly vs. every-3-week paclitaxel for ovarian cancer. N Engl J Med 374: 2602, 2016.

27. Dessai SB, Chakraborty S, Babu TV, Nayanar S, Bhattacharjee A Jones J, Balasubramanian S and Patil VM: Tolerance of weekly metronomic paclitaxel and carboplatin as neoadjuvant chemotherapy in advanced ovarian cancer patients who are unlikely to tolerate 3 weekly paclitaxel and carboplatin. South Asian J Cancer 5: 63-66, 2016.

28. Perkins CL, Fang G, Kim CN and Bhalla KN: The role of Apaf-1, caspase-9, and bid proteins in etoposide- or paclitaxel-induced mitochondrial events during apoptosis. Cancer Res 60: 1645-1653, 2000.

29. Pan J, Xu G and Yeung SC: Cytochrome c release is upstream to activation of caspase-9, caspase-8, and caspase-3 in the enhanced apoptosis of anaplastic thyroid cancer cells induced by manumycin and paclitaxel. J Clin Endocrinol Metab 86: 4731-4740, 2001.

30. Oktem M, Dilek TU, Guner H and Tiras MB: The effect of recombinant GM-CSF on IL-6 and TNF-alpha levels in epithelial ovarian cancer patients who received paclitaxel and cisplatinum: Preliminary results. Eur J Gynaecol Oncol 25: 478-480, 2004.

31. Suyama H, Igishi T, Sano H, Matsumoto S, Shigeoka Y, Nakanishi H, Endo M, Burioka N, Hitsuda Y and Shimizu E: ERK activation and subsequent RB phosphorylation are important determinants of the sensitivity to paclitaxel in lung adenocarcinoma cells. Int J Oncol 24: 1499-1504, 2004.

32. Atjanasuppat K, Lirdprapamongkol K, Jantaree P and Svasti J: Non-adherent culture induces paclitaxel resistance in H460 lung cancer cells via ERK-mediated up-regulation of $\beta$ IVa-tubulin. Biochem Biophys Res Commun 466: 493-498, 2015.

33. Wu G, Qin XQ, Guo JJ, Li TY and Chen JH: AKT/ERK activation is associated with gastric cancer cell resistance to paclitaxel. Int J Clin Exp Pathol 7: 1449-1458, 2014.

34. Cheng YJ, Lee CH, Lin YP, Huang JY, Su CC, Chang WT and Yang BC: Caspase-3 enhances lung metastasis and cell migration in a protease-independent mechanism through the ERK pathway. Int J Cancer 123: 1278-1285, 2008.

This work is licensed under a Creative Commons Attribution-NonCommercial-NoDerivatives 4.0 International (CC BY-NC-ND 4.0) License. 